\title{
Awareness on nutrition among the pregnant women at a female hospital in Jessore, Bangladesh
}

\author{
MM Rahman
}

\begin{abstract}
The study assessed the awareness on nutrition among the pregnant women at Female Ad-din Hospital in Jessore, Bangladesh from December 2011 to March 2012. One hundred seventy seven newly delivered mothers were randomly selected for interview on nutrition during pregnancy. Detailed history on food intake by the mothers during pregnancy period were recorded and analyzed. Many of the respondents did not take adequate amount of rice, vegetables, pulses, protein, vitamins, iron or minerals during pregnancy. This was due to that many of the pregnant mothers were not aware about increased food intake need during pregnancy period. Considering insufficient awareness in respect to nutritional behaviors among females of reproductive age, pregnant women and those who plan for pregnancy should be educated about adequate diet.
\end{abstract}

Key words: Awareness, Nutrition, Pregnant Women, Bangladesh

\section{Introduction}

Adequate nutrition during pregnancy is essential for healthy mother and healthy baby. Birth weight is a good indicator of community health. It is a good tool for assessing health care delivery at community level as well as the status of the mothers prenatally and during pregnancy. It is considered a predictor of neonatal viability and has an inverse relationship with neonatal perinatal mortality which is exceptionally high in Asian countries. It also remains an important factor affecting infant and child mortality. The nutrition of a woman during pregnancy does not deviate much from the principles of rational nutrition for each of us. In association with the new life conceived and developing her womb, the energetic demand increases by approximately $300 \mathrm{kcl}$ during $2^{\text {nd }}$ and $3^{\text {rd }}$ trimester of pregnancy. ${ }^{1} \mathrm{An}$ increase in body weight in pregnancy was specified in 1990 by the American Health Institute, updated in 2009 , based on tendencies observed in body weight increase during pregnancy from beginning of the 1900s among pregnant women in the USA. ${ }^{2}$ In addition, the Committee on nutritional status during pregnancy and lactation allows in the case of multiple pregnancies a higher increase in body weight during the period of pregnancy. The increase in body weight during pregnancy is usually about $17-25 \mathrm{~kg}$ for women with normal body weight, 14-23 
$\mathrm{kg}$ for women with overweight and $11-19 \mathrm{~kg}$ for obese women. Such standards concern women bearing babies from multiple pregnancies with the baby weight over 2.5 $\mathrm{kg}$ between 37-42 weeks of pregnancy with normal weight maturity. While specifying the energetic demands of the organism of a pregnant women attention should be paid to such elements as e.g., her age, current nutritional status (obesity, under weight), physical effort or type of occupation performed. The daily number of portions of food products from individual groups suggested for pregnant women is presented in the nutritional food pyramid for women by the World Health Organization (WHO). ${ }^{3}$ Pregnant women should select products for their daily diet in such a way that it contains 9 servings of products containing carbohydrates, 4 servings of vegetables, 3 servings of fruits, and 3 servings containing dairy products, meat, fish, eggs and nuts and sporadically, fats and sweets.

According to the results of the recommendations by the $\mathrm{WHO}$, the rational diet of pregnant women should supply an adequate amount of energy, and should also include indispensable nutrients (i.e. proteins, fats, carbohydrates, minerals and vitamins) in appropriate amounts and proportions. An adequate content of good beverage in the every day diet is also important. Dietary errors of pregnant women occur when the daily menu does not fully cover an increased demand for individual nutrients. Sometimes, in meals consumed daily by the women, there is an insufficient amount of microelements and vitamins. However, it should be remembered that also an excess amount of food products consumed and an additional supplementation of vitamins and microelements may be hazardous for the mother and the developing foetus. Approximately $60 \%$ of the total amount of daily demand for proteins is those of animal origin, the main source of which is milk and dairy products, as well as meat, poultry and fish. The remaining $40 \%$ should come from valuable plant proteins, e.g. pulses. Pregnant women also have an increased demand for some indispensable fatty acidslinoleic acid and alphalinoleic acid. ${ }^{3}$ The contents of carbohydrates in the daily diet of expectant mothers should be $400 \mathrm{gm}$ daily. On average during pregnancy an excessive amount of sweets and confectionery should not be consumed, while the consumption of complex carbohydrates is recommended. According to the recommendations by the $\mathrm{WHO}$, the supply of over $400 \mathrm{gm} /$ day of fruits and vegetables covers the daily demand for vitamins and minerals. ${ }^{3}$ The objective of the study was to analyze the nutritional behaviors (knowledge, attitude and practice) among pregnant women at a hospital in Jessore, Bangladesh.

\section{Methods}

This is a cross sectional descriptive type study. The general objective was to evaluating awareness (knowledge, attitude and practice) of the respondents on nutrition among pregnant women. The study population was the mothers who delivered their baby in a delivery unit of Ad-din hospital, Jessore, west region of Bangladesh. The sample size is 177 mothers who delivered their baby in this hospital. Data were collected using a questionnaire by asking questions about food taking habit during pregnancy.

\section{Results}

The total number of pregnant mothers in the present study was 177 . Out of those, 36 $(20.3 \%)$ pregnant mothers took normal or less amount of food during their pregnancy (Table 1). Tables 2 shows that 35 (19.8\%), $24(13.0 \%), 19$ (10.7\%) and 6 (3.4\%) pregnant mothers during their pregnancy took normal or less amount of rice, vegetables, pulses and animal protein, respectively. Tables 3 shows that $51(28.8 \%)$ and $31(17.5 \%)$ of pregnant mothers during their pregnancy did not take vitamins and iron, respectively.

\section{Discussion}

British researchers reported that an increased intake was observed for consumption of white bread, breakfast cereals, cakes, processed meat, chips, fruits, 
Table 1. Trend of overall food intake by mothers during pregnancy

\begin{tabular}{lcr}
\hline Intake trend & Number of pregnant mothers & $\%$ \\
\hline Normal or less intake & 36 & 20.32 \\
Increased intake & 141 & 79.66 \\
\hline Total & 177 & 100.00
\end{tabular}

Table 2. Intake trend for rice, vegetables, pulses and animal protein by mothers during pregnancy

\begin{tabular}{llcr}
\hline Item & Intake trend & Number of pregnant mothers & $\%$ \\
\hline \multirow{2}{*}{ Rice } & Normal or less intake & 35 & 19.77 \\
& Increased intake & 142 & 80.23 \\
\hline \multirow{2}{*}{ Vegetables } & Normal or less intake & 24 & 12.99 \\
& Increased intake & 53 & 87.01 \\
\hline \multirow{2}{*}{ Pulses } & Normal or less intake & 19 & 10.73 \\
& Increased intake & 158 & 89.27 \\
\hline \multirow{2}{*}{ Animal protein } & Normal or less intake & 6 & 3.39 \\
& Increased intake & 171 & 96.61 \\
\hline
\end{tabular}

Table 3. Vitamin and iron supplementations by mothers during pregnancy

\begin{tabular}{llcc}
\hline Item & Intake trend & Number of pregnant mothers & $\%$ \\
\hline \multirow{2}{*}{ Vitamins } & Normal or less intake & 35 & 19.77 \\
& Increased intake & 142 & 80.23 \\
\hline \multirow{2}{*}{ Iron } & Normal or less intake & 24 & 12.99 \\
& Increased intake & 153 & 87.01 \\
\hline
\end{tabular}

and fruit juices, sweets, hot chocolate, cream, cheese, polysaturated fats and oils, lettuce, red meat and non-alcoholic beverages; whereas a decreased intake was noted for consumption of rice, pasta, giblets, vegetables, nuts, diet coke, coffee, tea, boiled potatoes and crackers during pregnancy. ${ }^{4}$

The present study revealed that many of the pregnant mothers were not aware about increased food intake need during pregnancy period. Many of them took normal or less amount of rice, vegetables, pulses and animal protein. A large number of pregnant mothers during their pregnancy did not take vitamins and iron. Among pregnant women examined by Pinto et al, the mean daily used antenatal test of fetal well-being is cardiotocography (CTG). CTG is the graphic presentation of the fetal heart activity and the uterine contraction to detect fetal 
consumption of milk and dairy products in pregnancy was nearly twice as high as before pregnancy. ${ }^{5}$ The consumption of fats, breadstuff, fruit and soups also increased considerably in pregnancy, while consumption of eggs, red meat, pasta, potatoes, fast food products, alcoholic beverages, coffee and tea significantly decreased. ${ }^{5}$ In the study, two period points were analyzed and no statistically significant difference was noted for consumption of fish, vegetables, sweets, non-alcoholic beverages and fruit juices. The daily number of meals in pregnancy increased up to 5 meals, 54\% before pregnancy to $72 \% .^{5}$ In a study by Bojar et al, pregnant women apart from increasing the amount of product consumption after becoming pregnant change the proportions. ${ }^{6}$ A decrease was observed in the amount of fruits consumed with relation to the remaining food products, whereas an increase was noted in case of meat, vegetables and cereal products in the diet applied. ${ }^{6}$ Higher percentages of negative changes among the respondents living in the urban areas were noted concerning the amount and frequency of consumption of vegetables and fruits when compared to pregnant rural women. ${ }^{6}$ Studies conducted among pregnant women in Italy confirmed that the nutrition habit of expectant women during second trimester of pregnancy until delivery, both qualitative and quantitative, was non-rational, and knowledge of this problem was poor. ${ }^{7,8}$ The women consumed excessive amount of highly caloric products and saturated fats, while their intake of dairy products and vegetables was insufficient. Food products consumed by these pregnant women increased the risk of occurrence of vitamin deficiency, especially folic acid and riboflavin. Spanish researchers noted that the consumption of food products and microelements in their country differs slightly before conception and during pregnancy. ${ }^{9}$ During pregnancy, the consumption of milk, fruits and vegetables increased, while the intake of sugar and alcohol decreased. A Finish study confirmed that pregnant women consumed more microelements (by 30-40\%) than recommended by the WHO. ${ }^{10}$ An American study shows that the diet of a considerable part of the women population of reproductive age does not cover the demand for microelements and vitamins according to the established recommendations. ${ }^{11}$ Rural Chinese women during their third trimester of pregnancy consumed an insufficient amount of the majority of nutrients in their diet, including those especially important during pregnancy, such as iron, zinc, riboflavin and folic acid. ${ }^{8}$

\section{Conclusion}

Many of the respondents in the study did not take adequate amount of rice, vegetables, pulses, protein, vitamins, iron or minerals during pregnancy. More than $70 \%$ of pregnant women of the study were aware about nutrition during pregnancy and the rest women were not aware about increased food intake need during pregnancy. Considering insufficient awareness in respect to nutritional behaviors among females of reproductive age, pregnant women and those who plan for pregnancy should be educated about adequate diet.

\section{References}

1. Szostak-Wegierek $D$ and Cichocka $A$. Zywienie Kobiet w Ciazy [Nutritional advices for pregnant women]. PZWL, Warsaw: 2005.

2. Rasmussen KM and Yaktine AL. Weight gain during pregnancy: reexamining the guidelines, Food and Nutrition Board (FNB), Board on Children, Youth and Families (BOCYF). The National Academies Press, Washington, DC 2009. Available from: http://www.nap.edu.

3. Healthy Food and Nutrition for women and their Families. Training Course for Health Professionals. WHO. Regional office for Europe. Regional office for Unicef, Geneva, Central and Eastern Europe and the Commonwealth of Independent States and the Baltics.

4. Crozier SR, Robinson SM, Godfery KM, Cooper C, Inskip HM. Women's dietary change little from before to during pregnancy. J Nutr 2009;139:1956-63.

5. Pinto $E$, Barros $H$, Silvia I. Dietary intake and nutritional adquecy prior to conception and during pregnancy: a 
follow-up study in the north of Portugal. Public Health Nutr 2009;12(7):922-31.

6. Bojar I, Wdowiak L, Humeniuk E, Blaziak $P$. Change in the quality of diet during pregnancy in comparison with $\mathrm{WHO}$ and EU recommendations- environmental and sociodemopraphic conditions. Ann Agric Environ Med 2006;13(2):281-6.

7. Fidanza $A A$ and Fidanza R: A nutrition study involving a group of pregnant women in Assisi, Italy. Part 1: Anthropometry, dietary intake and nutrition knowledge, practices and attitudes. Int $\mathrm{J}$ Vitam Nutr Res 1986;56:373-80.

8. Fidanza AA, Simonetti MS, Cucchia LM. A nutrition study involving of a group of pregnant women in Assisi, Italy. Part 2: Detrmination of vitamin nutriture. Int $\mathrm{J}$
Vitam Nutr Res 1986;56:381-6.

9. Arija V, Cuco G, Vila J, Iranzo R, Fernandez-Ballart J. Food consumption, dietary habits and nutritional status of the population of Reus: Follow-up from preconception throughout pregnancy and after birth. Med Clin (Barc) 2004;123:511.

10. Erkkola M, Karppinen M, Javanainen J, Rasaneu L, Virtnen SM. Validity and roducibility of food frequency questionnaire for pregnant Finnish women. Am J Epidemiol 2001;154:46676.

11. Bodnar LM and Siega-Riz AM. A Diet Quality Index for Pregnancy detects variation in diet and differences by sociodemographic factors. Public Health Nutr 2002;5:801-9. 